\title{
SDSS superclusters: morphology and galaxy content ${ }^{\star}$
}

\author{
M. Einasto ${ }^{1}$, H. Lietzen ${ }^{1,2,3}$, E. Tempel ${ }^{1,4}$, M. Gramann ${ }^{1}$, L. J. Liivamägi ${ }^{1,5}$, and J. Einasto ${ }^{1,6,7}$ \\ 1 Tartu Observatory, 61602 Tõravere, Estonia \\ 2 Instituto de Astrofísica de Canarias, 38200 La Laguna, Tenerife, Spain \\ 3 Universidad de La Laguna, Dept. Astrofísica, 38206 La Laguna, Tenerife, Spain \\ ${ }^{4}$ National Institute of Chemical Physics and Biophysics, Rävala pst 10, 10143 Tallinn, Estonia \\ 5 Institute of Physics, Tartu University, Tähe 4, 51010 Tartu, Estonia \\ ${ }^{6}$ Estonian Academy of Sciences, 10130 Tallinn, Estonia \\ 7 ICRANet, Piazza della Repubblica 10, 65122 Pescara, Italy
}

Received 22 November 2013 / Accepted 31 December 2013

\section{ABSTRACT}

\begin{abstract}
Context. Understanding the formation, evolution and present-day properties of the cosmic web and objects forming it is an important task in cosmology.

Aims. We compare the galaxy populations in superclusters of different morphology in the nearby Universe $\left(180 h^{-1} \mathrm{Mpc} \leq d \leq\right.$ $270 \mathrm{~h}^{-1} \mathrm{Mpc}$ ) to see whether the inner structure and overall morphology of superclusters are important in shaping galaxy properties in superclusters.

Methods. We find supercluster morphology with Minkowski functionals and analyse the probability density distributions of colours, morphological types, stellar masses, star formation rate (SFR) of galaxies, and the peculiar velocities of the main galaxies in groups in superclusters of filament and spider types, and in the field. We test the statistical significance of the results with the KS test.

Results. The fraction of red, early-type, low SFR galaxies in filament-type superclusters is higher than in spider-type superclusters; in low-density global environments their fraction is lower than in superclusters. In all environments the fraction of red, high stellar mass, and low SFR galaxies in rich groups is higher than in poor groups. In superclusters of spider morphology red, high SFR galaxies have higher stellar masses than in filament-type superclusters. Groups of equal richness host galaxies with larger stellar masses, a larger fraction of early-type and red galaxies, and a higher fraction of low SFR galaxies, if they are located in superclusters of filament morphology. The peculiar velocities of the main galaxies in groups from superclusters of filament morphology are higher than in those of spider morphology. Groups with higher peculiar velocities of their main galaxies in filament-type superclusters are located in higher density environment than those with low peculiar velocities. There are significant differences between galaxy populations of the individual richest superclusters.

Conclusions. Both local (group) and global (supercluster) environments and even supercluster morphology play an important role in the formation and evolution of galaxies. Differences in the inner structure of superclusters of filament and spider morphology and the dynamical state of galaxy groups in them may lead to the differences found in our study.
\end{abstract}

Key words. large-scale structure of Universe - galaxies: general - galaxies: clusters: general

\section{Introduction}

The large scale structure of the Universe is formed by a hierarchy of galaxy systems from isolated galaxies to groups, clusters, and superclusters of galaxies. Galaxy superclusters, environments for galaxies, and groups and clusters of galaxies, are the largest relatively isolated systems in the Universe are galaxy superclusters (de Vaucouleurs 1956; Jõeveer et al. 1978; Einasto et al. 1984, 1994; Zucca et al. 1993). To understand the role of small-scale (group) and large-scale (supercluster) environments in galaxy formation and evolution, we need to study the properties of superclusters and their galaxy and group populations together.

With the advent of deep surveys covering large areas in the sky it became possible to determine the parameters of a large number of superclusters, and to study in detail the galaxy and group populations in them. These studies have revealed that in richer superclusters groups and clusters of galaxies are also richer, and they contain a larger fraction of red, non-star forming galaxies than poor superclusters (Einasto et al. 2007, 2007a).

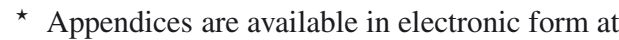
http: //www . aanda.org
Early studies of galaxy environment showed that environments at the group and cluster scale affects the morphology of galaxies, early-type, more luminous galaxies are located in higher density environments than late-type, fainter galaxies (Einasto et al. 1974; Dressler 1980; Postman \& Geller 1984; Einasto \& Einasto 1987; Hamilton 1988; Einasto 1991). The environment affects the properties of galaxies and groups of galaxies even at larger scales, up to $10-15 h^{-1} \mathrm{Mpc}$ (Einasto \& Einasto 1987; Mo et al. 1992; Einasto et al. 2003a,b; Skibba 2009; Tempel et al. 2011). In addition, groups of the same richness contain a larger fraction of red, non-star-forming galaxies if they are located in rich superclusters compared to groups in poor superclusters or in low-density large-scale environments outside of superclusters (Einasto et al. 2008; Lietzen et al. 2012). Luparello et al. (2013) showed that the stellar population of galaxies in groups in superstructures is systematically older, and groups themselves are larger and have higher velocity dispersions than groups which do not belong to superstructures.

The morphology of superclusters have been studied, for example, by Costa-Duarte et al. (2011) and Einasto et al. (2007b). Galaxy superclusters have complex inner structures that can be quantified with morphological descriptors as Minkowski 
functionals. Einasto et al. (2007b, 2011a) found in the wide morphological variety of superclusters two main types of superclusters: filaments and spiders. In filaments high-density core(s) of superclusters are connected by a small number of galaxy chains (filaments). In spiders there are many galaxy chains between high-density cores in superclusters. Poor spider-type superclusters are similar to our Local supercluster with one rich cluster and filaments of galaxies and poor groups of galaxies around it (Einasto et al. 2007b).

Einasto et al. (2012a) analysed the structure of rich galaxy clusters in superclusters of different morphologies and showed that clusters in superclusters of spider morphology have higher probabilities of having substructure and their main galaxies have higher peculiar velocities than clusters in superclusters of filament morphology.

In the present study we continue our analysis of superclusters of different morphology, studying their galaxy and group populations, to understand better how the large-scale (supercluster) environment affects the properties of galaxies and groups residing in them. For comparison we also analyse galaxy content and group properties in low-density global environment (field).

In Sect. 2 we describe the data about galaxies, groups, and superclusters used in this paper, in Sect. 3 we compare the galaxy content and the peculiar velocities of the main galaxies in groups in superclusters of different type, and in the field. We discuss the results and draw conclusions in Sect. 4.

We assume the standard cosmological parameters: the Hubble parameter $H_{0}=100 \mathrm{~h} \mathrm{~km} \mathrm{~s}^{-1} \mathrm{Mpc}^{-1}$, the matter density $\Omega_{\mathrm{m}}=0.27$, and the dark energy density $\Omega_{\Lambda}=0.73$ (Komatsu et al. 2011).

\section{Data}

\subsection{Galaxy, group, and supercluster data}

We use the MAIN sample of the 8th data release of the Sloan Digital Sky Survey (SDSS DR8; Aihara et al. 2011) with the apparent Galactic extinction corrected $r$ magnitudes $r \leq 17.77$, and the redshifts $0.009 \leq z \leq 0.200$, in total 576493 galaxies. We corrected the redshifts of galaxies for the motion relative to the CMB and computed the co-moving distances (Martínez \& Saar 2002) of galaxies.

Galaxy groups were determined using the Friends-ofFriends cluster analysis method introduced in cosmology by Zeldovich et al. (1982) and Huchra \& Geller (1982). A galaxy belongs to a group of galaxies if this galaxy has at least one group member galaxy closer than a linking length. In a fluxlimited sample the density of galaxies slowly decreases with distance. To take this selection effect into account properly when constructing a group catalogue from a flux-limited sample, we rescaled the linking length with distance, calibrating the scaling relation by observed groups (see Tago et al. 2008, 2010, for details). As a result, the maximum sizes in the sky projection and the velocity dispersions of our groups are similar at all distances. Our initial sample is chosen in the distance interval $120 h^{-1} \mathrm{Mpc} \leq d \leq 340 h^{-1} \mathrm{Mpc}$ (the redshift range $0.04<z<0.09$ ) where the selection effects are the smallest (we discuss the selection effects in detail in Tago et al. 2010; Einasto et al. 2012a). The details of data reduction and the description of the group catalogue can be found in Tempel et al. (2012).

We calculate the galaxy luminosity density field to reconstruct the underlying luminosity distribution and to determine superclusters (extended systems of galaxies) in the luminosity density field at smoothing length $8 h^{-1} \mathrm{Mpc}$ using $B_{3}$ spline kernel (see Einasto et al. 2007b, for comparison between $B_{3}$ and Gaussian kernel). We created a set of density contours by choosing a density thresholds and defined connected volumes above a certain density threshold as superclusters. In order to choose a proper density level to determine individual superclusters, we analysed the properties of the density field superclusters at a series of density levels. As a result we used the density level $D_{8}=5.0$ (in units of mean density, $\ell_{\text {mean }}=1.65 \times 10^{-2} \frac{10^{10} h^{-2} L_{\odot}}{\left(h^{-1} \mathrm{Mpc}\right)^{3}}$ ) to determine individual superclusters (Liivamägi et al. 2012). At this density level superclusters in the richest chains of superclusters in the volume under study still form separate systems; at lower density levels they join into huge percolating systems. At higher threshold density levels superclusters are smaller and their number decreases.

The morphology of all superclusters used in this study was determined in Einasto et al. (2012a) applying Minkowski functionals. For a given surface the four Minkowski functionals are proportional to the enclosed volume $V$, the area of the surface $S$, the integrated mean curvature $C$, and the integrated Gaussian curvature $\chi$ (see Appendix B and Einasto et al. 2007b, 2011a, for details and references). The overall morphology of a supercluster is described by the shapefinders $K_{1}$ (planarity) and $K_{2}$ (filamentarity), and their ratio, $K_{1} / K_{2}$ (the shape parameter), calculated using the first three Minkowski functionals. The lower the value of the shape parameter, the more elongated a supercluster is. The maximum value of the fourth Minkowski functional $V_{3}$ characterises the inner structure of the superclusters. The higher the value of $V_{3}$, the more complicated the inner morphology of a supercluster is (Einasto et al. 2007b, 2011b). Superclusters were classified as filaments and spiders on the basis of their morphological information and visual appearance. Superclusters show wide morphological variety in which Einasto et al. (2011a) determined four main morphological types: spiders, multispiders, filaments, and multibranching filaments. Spiders and multispiders are systems of one or several high-density clumps with a number of outgoing filaments connecting them. Filaments and multibranching filaments are superclusters with filament-like main bodies that connect clusters in superclusters. For simplicity, in this study we classify superclusters as spiders and filaments. In Appendix B we show an examples of filament and spider-type superclusters.

The description of the supercluster catalogues is given in Liivamägi et al. (2012), the details of supercluster morphology are given in Einasto et al. (2007b, 2011a).

Figure 1 presents the distribution of rich galaxy clusters in superclusters of filament and spider morphology, as well as from the field in cartesian coordinates $x, y$, and $z$ defined as in Park et al. (2007) and in Liivamägi et al. (2012):

$$
\begin{aligned}
& x=-\mathrm{d} \sin \lambda, \\
& y=\mathrm{d} \cos \lambda \cos \eta, \\
& z=\mathrm{d} \cos \lambda \sin \eta,
\end{aligned}
$$

where $d$ is the finger of god corrected comoving distance, and $\lambda$ and $\eta$ are the SDSS survey coordinates.

Up to distances of about $180 h^{-1} \mathrm{Mpc}$ the SDSS survey crosses the void region between the Hercules supercluster and the chain of rich superclusters farther away (the Bootes void, Kirshner et al. 1981). Two chains of galaxies, groups, and superclusters cross this void. At the distance interval of about $180 h^{-1} \mathrm{Mpc} \leq d \leq 270 h^{-1} \mathrm{Mpc}$ the survey crosses a number of rich superclusters, including the Sloan Great Wall (detailed description of the large-scale distribution of superclusters can be 

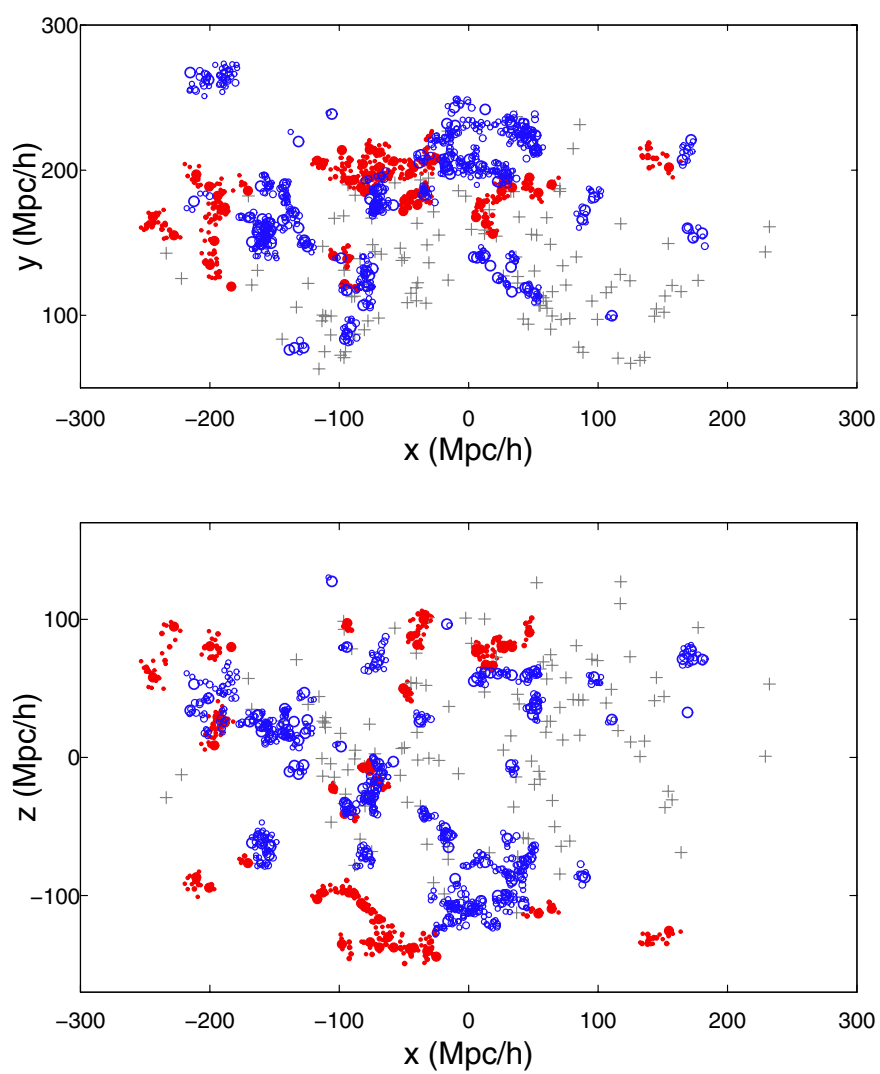

Fig. 1. Distribution of galaxy groups with at least four member galaxies in superclusters in $x, y$, and $z$ coordinates. Red filled circles correspond to groups in filament-type superclusters. Blue empty circles denote groups in spider-type superclusters. Grey crosses denote groups with at least 30 member galaxies from the field.

found in Einasto et al. 2011b,a). At distances $d>270 h^{-1} \mathrm{Mpc}$ there is another void region, and the frequency of superclusters in this region is very low. This is also seen in Fig. 2 where we plot the distributions of distances to red and blue galaxies divided by their colour index $g-r=0.7$ (see Sect. 2.2) in superclusters and in the field.

We have different distance intervals with different largescale distributions and richness of systems. From these we analysed data from two regions: a void region with poor systems, $120 h^{-1} \mathrm{Mpc} \leq d \leq 180 h^{-1} \mathrm{Mpc}$ (near sample), and a supercluster region with rich systems of galaxies $180 h^{-1} \mathrm{Mpc} \leq d \leq$ $270 h^{-1} \mathrm{Mpc}$ (distant sample). Among the 50 superclusters studied, 35 are of spider morphology and 15 of filament morphology. The supercluster data are given in Table A.1. We do not use data for very poor superclusters without any rich clusters whose morphology is difficult to determine (for details we refer to Einasto et al. 2012a).

Our data are based on a flux-limited sample of galaxies in which absolute magnitude limit increases with distance, introducing selection effects: there are relatively more red galaxies at great distances (this can also be seen in Fig. 2). In order to avoid this selection effect we used volume-limited samples with $M_{r} \leq-18.5$ in near samples, and $M_{r} \leq-19.5$ in distant samples. The numbers of galaxies in near and distant samples of superclusters are given in Table 1. The number of galaxies in filament-type superclusters from near sample is small. Our calculations showed that the results for the near and distant samples are similar, but the statistics of the near sample are not very reliable, therefore in the paper we present our results for the distant sample only.
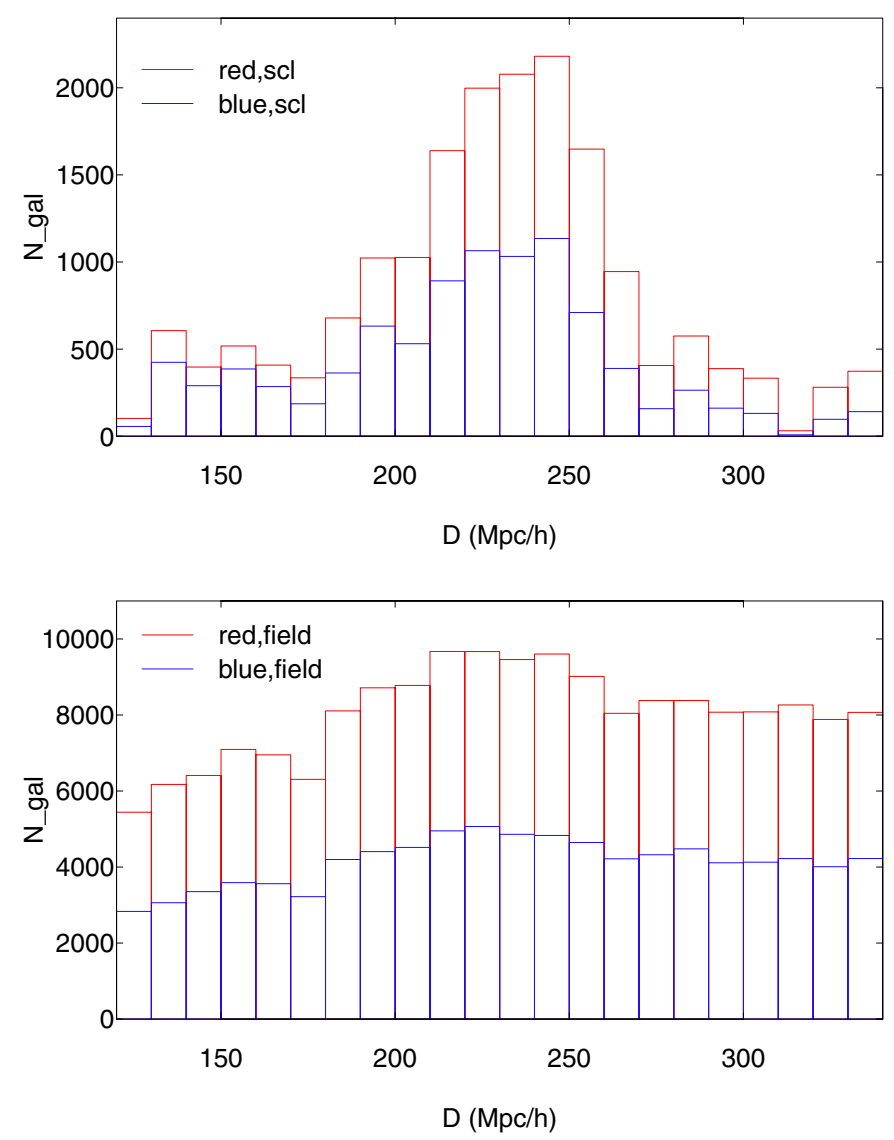

Fig. 2. Distribution of distances of red and blue galaxies (red and blue lines, respectively) in superclusters (upper panel) and in the field (lower panel).

Table 1. Number of galaxies in filament and spider-type superclusters and in field for flux- and volume-limited samples.

\begin{tabular}{lcccc}
\hline \hline & \multicolumn{2}{c}{$120-180 h^{-1} \mathrm{Mpc}$} & \multicolumn{2}{c}{$180-270 h^{-1} \mathrm{Mpc}$} \\
Sample & flux-lim & vol-lim & flux-lim & vol-lim \\
\hline Filaments & 517 & 500 & 8102 & 6664 \\
Spiders & 3497 & 3024 & 12030 & 9211 \\
Field & 58712 & 50643 & 123230 & 93795 \\
\hline
\end{tabular}

\subsection{Galaxy properties}

In the present paper we use the $g-r$ colours of galaxies and their absolute magnitudes in the $r$-band $M_{r}$. The absolute magnitudes of individual galaxies are computed according to the formula

$M_{r}=m_{r}-25-5 \log _{10}\left(d_{L}\right)-K$,

where $d_{L}$ is the luminosity distance in units of $h^{-1} \mathrm{Mpc}$ and $K$ is the $k+e$-correction. The $k$-corrections were calculated with the KCORRECT (v4_2) algorithm (Blanton \& Roweis 2007) and the evolution corrections have been calibrated according to Blanton et al. (2003). We use $M_{\odot}=4.53$ (in $r$-filter). All of our magnitudes and colours correspond to the rest-frame at redshift $z=0$. The value $g-r=0.7$ is used to separate red and blue galaxies, red galaxies having $g-r \geq 0.7$. We used the morphology classification by Huertas-Company et al. (2011) which gives for each galaxy a probability of being early and late type, $p_{\mathrm{e}}$ and $p_{\mathrm{l}}$, correspondingly. Approximately, we call a galaxy late type, if $p_{\mathrm{l}}>0.5$, and early type, if $p_{\mathrm{e}}>0.5$.

To study the stellar masses $\log M_{\mathrm{S}}$ and star formation rates (SFRs), we use the MPA-JHU spectroscopic catalogue 
(Tremonti et al. 2004; Brinchmann et al. 2004). In this catalogue the different properties of galaxies are obtained by fitting SDSS photometry and spectra with the stellar population synthesis models developed by Bruzual \& Charlot (2003). The stellar masses of galaxies are derived in the manner described by Kauffmann et al. (2003). For the DR8 data the stellar masses are estimated from the galaxy photometry (rather than the spectral indices $D_{n} 4000$ and $H_{\delta}$ used by Kauffmann et al. 2003). The SFRs are computed using the photometry and emission lines as described by Brinchmann et al. (2004) and Salim et al. (2007). For active galactic nuclei and galaxies with weak emission lines, SFRs are estimated from the photometry. For star-forming galaxies with strong emission lines, the SFRs are estimated by fitting different emission lines in the galaxy spectrum $\left(H_{\alpha}, H_{\beta}\right.$, [O III] 5007, [N II] 6584, [O II] 3727, [S II] 6716). The stellar masses and SFRs are taken from the SDSS CAS database.

In the group catalogue the main galaxy of a group is defined as the most luminous galaxy in the $r$-band. We also use this definition in the present paper. In virialized clusters galaxies follow the cluster potential well, and so we would expect that the main galaxies in clusters lie at the centres of groups and have low peculiar velocities (Ostriker \& Tremaine 1975). Therefore the peculiar velocity of the main galaxies in clusters is also an indication of the dynamical state of the cluster (Coziol et al. 2009; Einasto et al. 2012b). We calculate the peculiar velocities of the main galaxies, $\left|v_{\text {pec }}\right|$, and compare these velocities for groups in superclusters of different morphology, and in the field.

\section{Results}

\subsection{Superclusters of different morphology}

We analyse galaxy populations in superclusters of different morphology, and in the field using probability density distributions which are calculated within the statistical package $\mathrm{R}$ environment using the "density" command in the "stats" package (Ihaka \& Gentleman 1996) ${ }^{1}$. In Tables in Appendix $C$ we present the values of quantiles of the parameters analysed in the paper. The distributions of colours, probabilities of being early or late type, stellar masses, and SFRs are bimodal or asymmetrical; the use of full distributions in the analysis and quantile values in tables is straightforward. To not to make tables too overcrowded we do not include errors to the tables; the statistical significance of the differences between different galaxy properties have been found using the full data (the integral distributions) and the Kolmogorov-Smirnov (KS) test. We give the $p_{\mathrm{KS}}$-values of the test, throughout the paper (for details of this approach we refer to Einasto et al. 2008). We consider that the differences between distributions are highly significant if the $p_{\mathrm{KS}}$ value (the estimated probability of rejecting the hypothesis that distributions are statistically similar) $p_{\mathrm{KS}} \leq 0.05$.

Figure 3 present the distributions of galaxy $g-r$ colours, probabilities of being late type $\left(p_{1}\right)$, stellar masses $\left(\log M_{\mathrm{S}}\right)$, and SFRs for galaxies from superclusters of filament and spider morphology, and from the field. We show the distributions of latetype galaxies only; the distributions of early-type galaxies show similar differences between galaxy populations $\left(p_{\mathrm{e}}=1-p_{1}\right)$. The values of quantiles of galaxy parameters are given in Table C.1.

This figure shows, first of all, that galaxy populations in superclusters and in the field are different, superclusters containing a larger fraction of red, early-type, high stellar mass, and low SFR galaxies than can be found in the field. These figures show that galaxy populations in superclusters of filament

\footnotetext{
1 http://www.r-project.org
}
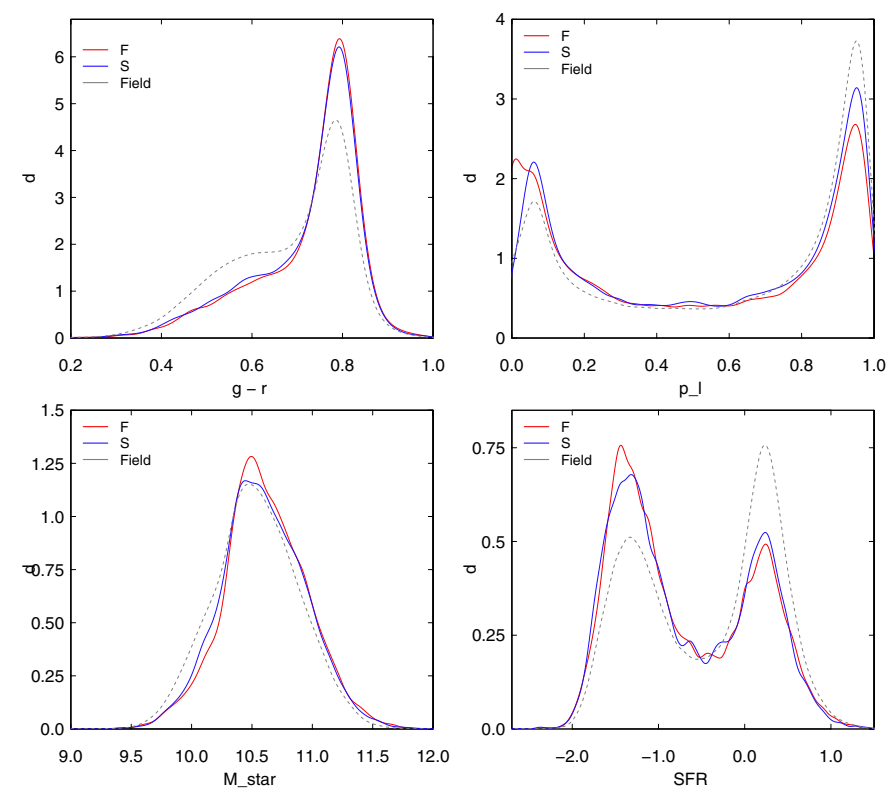

Fig. 3. Probability density distributions of galaxy $g-r$ colours (upper left panel), probabilities of being late type ( $p_{1}$, upper right panel), stellar masses $\left(\log M_{\mathrm{s}}\right.$ in $\log M_{\odot}$, lower left panel), and star formations rates (SFRs, in $\log M_{\odot} / \mathrm{yr}$ ) for galaxies in the superclusters of filament and spider morphology and in the field.

and spider morphology are also different, superclusters of filament morphology contain a larger fraction of red, early-type, low SFR galaxies than superclusters of spider morphology. All differences are statistically significant at very high confidence level $\left(p_{\mathrm{KS}}<0.001\right)$.

The distribution of stellar masses of galaxies in filament and spider-type superclusters shows that in filament-type superclusters there is some deficit of low stellar mass galaxies with $\log M_{\mathrm{S}}<10.25$, and relatively more galaxies of higher stellar mass than in superclusters of spider morphology. In Fig. 4 we show the probability density distributions of colours, types, and SFRs of high and low stellar mass galaxies in superclusters of filament and spider type. Table C.2 presents the values of quantiles of these parameters. As expected (see, for example, Blanton \& Moustakas 2009), among high stellar mass galaxies there are relatively more red, early-type, low SFR galaxies than among low stellar mass galaxies. Interestingly, this figure shows that low stellar mass galaxies in filament-type superclusters are mainly blue, but in spider-type superclusters there is relatively more red galaxies among them in comparison with filament-type superclusters. These differences are statistically highly significant, as also differences in galaxy types. Differences between SFRs of galaxies from filament and spider-type superclusters divided by stellar mass are low and statistically unsignificant, according to the KS test.

Next we divided galaxies by colours and SFRs as red, low SFR galaxies $(g-r \geq 0.7$, and $\log S F R<-0.5$, approximately $60 \%$ of all galaxies), blue, high SFR galaxies $(g-r<0.7$, and $\log S F R \geq-0.5,30 \%$ of all galaxies), and red, high SFR galaxies $(g-r \geq 0.7$, and $\log S F R \geq-0.5,10 \%$ of all galaxies). We plot the distributions of their stellar masses and types in Fig. 5, and give the values of quantiles of the stellar masses and types in Table C.3. This figure shows interesting feature: stellar masses of red, low SFR galaxies, and blue, high SFR galaxies in filament-type superclusters are higher than in spider-type superclusters, as were these masses in overall distributions (Fig. 3), 

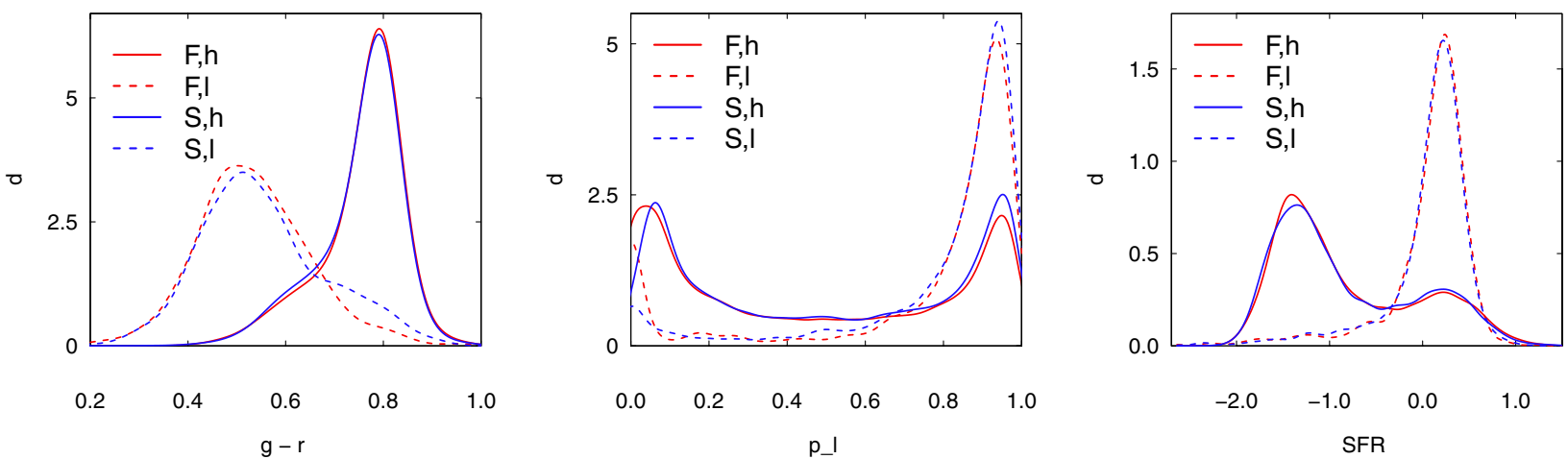

Fig. 4. Probability density distributions of galaxy $g-r$ colours (left panel), probabilities of being late type ( $p_{1}$, middle panel), and star formations rates (SFRs, in $\log M_{\odot} / \mathrm{yr}$, right panel) for high (h, $\log M_{\mathrm{s}} \geq 10.25$, solid lines) and low (l, $\log M_{\mathrm{s}}<10.25$, dashed lines) stellar mass galaxies in the superclusters of filament (F, red lines) and spider ( $\mathrm{S}$, blue lines) morphology.
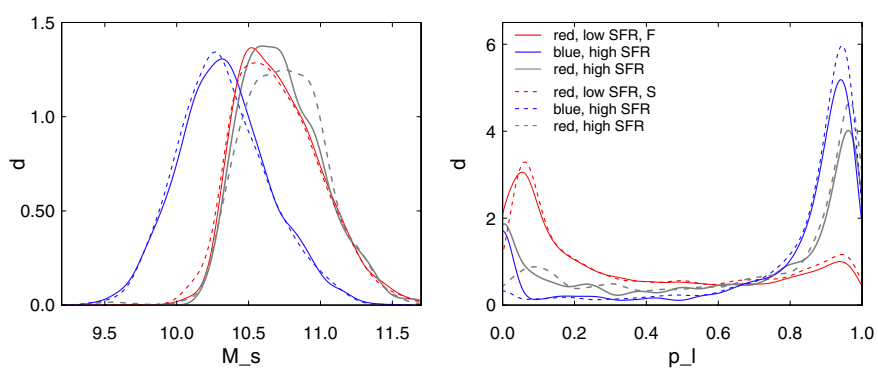

Fig. 5. Probability density distributions of stellar masses $\left(\log M_{\mathrm{s}}\right.$, in $\left.\log M_{\odot}\right)$, (left panel) and probabilities of being late type $\left(p_{1}\right.$, right panel), for red, low SFR galaxies (red lines), blue, high SFR galaxies (blue lines), and for red, high SFR galaxies (grey lines) in the superclusters of filament (solid lines) and spider (dashed lines) morphology. High SFR galaxies have $\log S F R \geq-0.5$, and low SFR galaxies have $\log S F R<-0.5$. Lines in the left panel denote the same populations as in the right panel.

but stellar masses of red, high SFR galaxies have higher values in spider-type superclusters. In filament-type superclusters their stellar masses are close to the stellar masses of red, low SFR galaxies, in spider-type superclusters their stellar masses are even higher than those of red, low SFR galaxies. These galaxies are mostly of late type. Thus this figure shows that high mass galaxies are red, even if they are of high SFR and of late type, and there is a difference in their masses in different types of superclusters. KS test shows that the differences between stellar masses and types of high and low SFR galaxies from filament and spider-type superclusters are statistically highly significant for both high and low SFR red galaxies.

Figure 6 shows the distributions of environmental densities at smoothing lengths $1,2,4$, and $8 h^{-1} \mathrm{Mpc}$ in filament and spider-type superclusters. At all smoothing lengths in these distributions poor groups with $N_{\text {gal }} \leq 9$ dominate at low densities; higher values of densities correspond to richer groups. Figure 6 shows a small deficit of intermediate-value densities in filamenttype superclusters, the KS test shows that the differences are statistically significant at least $95 \%$ level. If densities in filamenttype superclusters were systematically higher than in spider-type superclusters then the differences between galaxy populations in these superclusters were related to the dependence of the galaxy morphology on the environmental density, but we see that this is not so. Inner morphology of superclusters is important.

We also calculated environmental densities around galaxies of different colour and SFRs. Figure 7 shows environmental
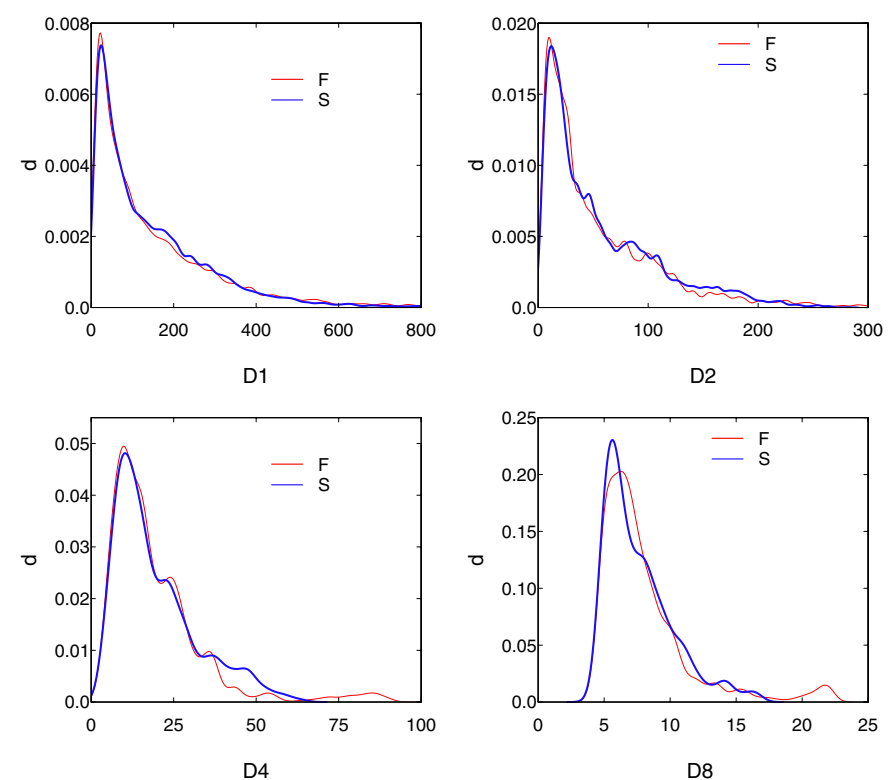

Fig. 6. Distributions of environmental densities at different smoothing lengths $\left(1,2,4\right.$, and $\left.8 h^{-1} \mathrm{Mpc}\right)$ in superclusters of filament (red lines) and spider (blue lines) morphology.

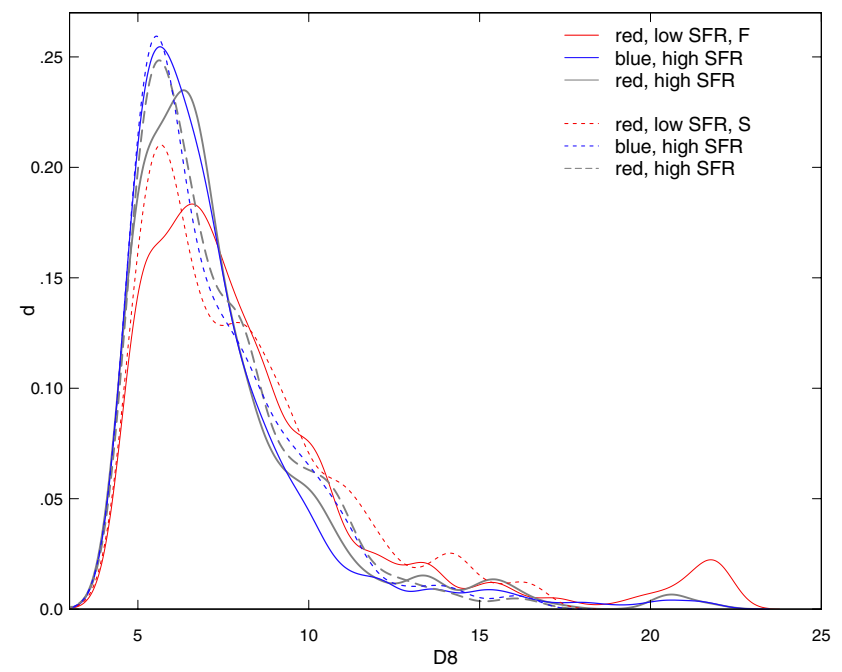

Fig. 7. Distributions of environmental density at smoothing length $8 h^{-1} \mathrm{Mpc}$ around red, low SFR galaxies (red lines), blue, high SFR galaxies (blue lines), and red, high SFR galaxies (grey lines) in superclusters of filament (solid lines) and spider (dashed lines) morphology. 
Table 2. Results of the KS test (the $p_{\mathrm{KS}}$-values): comparison of the galaxy properties in filament- and spider-type superclusters of different overall shape and richness.

\begin{tabular}{|c|c|c|c|c|}
\hline (1) & (2) & (3) & (4) & (5) \\
\hline \multicolumn{5}{|c|}{ More elongated and less elongated superclusters } \\
\hline & \multicolumn{2}{|c|}{ Filaments } & \multicolumn{2}{|c|}{ Spiders } \\
\hline & flux-lim & vol-lim & flux-lim & vol-lim \\
\hline & $p_{\mathrm{KS}}$ & $p_{\mathrm{KS}}$ & $p_{\mathrm{KS}}$ & $p_{\mathrm{KS}}$ \\
\hline$g-r$ & 0.515 & 0.735 & 0.331 & 0.107 \\
\hline$p_{1}$ & 0.099 & 0.535 & 0.003 & 0.011 \\
\hline $\log M_{\mathrm{s}}$ & $<1 e-5$ & 0.153 & 0.524 & 0.350 \\
\hline$S F R$ & 0.242 & 0.221 & 0.001 & 0.002 \\
\hline \multicolumn{5}{|c|}{ The richest superclusters } \\
\hline & \multicolumn{2}{|c|}{$\begin{array}{c}\text { Filaments } \\
\mathrm{SCl} 001, \mathrm{SCl} 027\end{array}$} & \multicolumn{2}{|c|}{$\begin{array}{c}\text { Spiders } \\
\mathrm{SCl} 019, \mathrm{SCl} 099\end{array}$} \\
\hline & $\begin{array}{c}\text { flux-lim } \\
p_{\mathrm{KS}}\end{array}$ & $\begin{array}{c}\text { vol-lim } \\
p_{\mathrm{KS}}\end{array}$ & $\begin{array}{c}\text { flux-lim } \\
p_{\mathrm{KS}}\end{array}$ & $\begin{array}{c}\text { vol-lim } \\
p_{\mathrm{KS}}\end{array}$ \\
\hline$g-r$ & $<1 e-5$ & $<1 e-5$ & 0.824 & 0.804 \\
\hline$p_{1}$ & $<1 e-5$ & $<1 e-5$ & 0.001 & 0.127 \\
\hline $\log M_{\mathrm{s}}$ & $<1 e-5$ & 0.063 & 0.012 & $<1 e-5$ \\
\hline SFR & 0.001 & 0.003 & 0.003 & 0.079 \\
\hline$D_{8}$ & $<1 e-5$ & $<1 e-5$ & $<1 e-5$ & $<1 e-5$ \\
\hline
\end{tabular}

Notes. Columns are as follows: 1: Galaxy properties. $g-r-$ colour index, $p_{1}-$ probability of being a late type, $\log M_{\mathrm{S}}-\log$ stellar mass, $S F R-\log S F R, D_{8}$ - environmental density at $8 h^{-1}$ Mpc scale smoothing scale; $2-5$ : the $p_{\mathrm{KS}}$-value of the test.

densities at $8 h^{-1}$ Mpc smoothing length around red, low SFR, blue, high SFR, and red, high SFR galaxies, analysed above. In this figure we see that blue, high SFR galaxies have somewhat lower environmental densities around them than red, low SFR galaxies in both type of superclusters. This is an evidence of a large-scale morphology-density relation. Environmental densities around red, low SFR galaxies in filament-type superclusters have higher values than in spider-type superclusters. Environmental densities around red, high SFR galaxies in spider-type superclusters are close to those for blue galaxies; in filament-type superclusters the densities around them have somewhat higher values. In filament-type superclusters some of these galaxies are located in a rather high-density environment. All differences are statistically highly significant.

Our sample contain several very rich superclusters. We analyse their galaxy populations separately (Sect. 3.3). To see whether our overall results are affected by their dominance we repeated all calculations excluding the richest systems from the sample. All our results remained the same, therefore neither the superclusters from the Sloan Great Wall, nor other very rich superclusters do not dominate in our results.

\subsection{Superclusters of different shape}

Superclusters can be divided into more and less elongated systems according to the value of their shape parameter $K_{1} / K_{2}$. The smaller the shape parameter, the more elongated a supercluster is. Superclusters with $K_{1} / K_{2}<0.5$ (more elongated systems) are called filament type, and those with $K_{1} / K_{2} \geq 0.5$ belong to pancake type being less elongated systems (Einasto et al. 2011a). Costa-Duarte et al. (2013) analysed stellar populations of galaxies in superclusters of different shape, using shape parameter to characterise the overall morphology of superclusters. Similarly, we use the shape parameter to divide superclusters of filament and spider types into sets of more elongated and less elongated systems and compare galaxy populations in them. The results are presented with the KS test in Table 2. For volume-limited
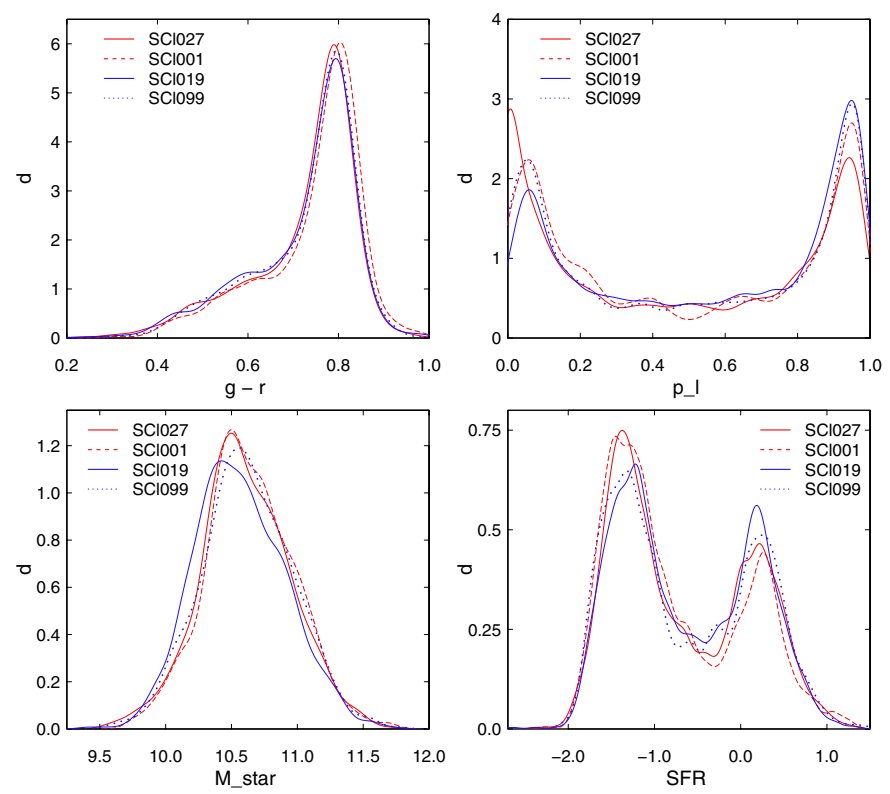

Fig. 8. Same as in Fig. 3 for the richest superclusters: filaments $\mathrm{SCl} 001$ and $\mathrm{SCl} 027$ (red dashed and solid lines), and spiders $\mathrm{SCl} 019$ and SCl 099 (blue solid and dashed lines).

samples galaxy populations in more elongated and less elongated superclusters of filament morphology are statistically similar. KS test shows that galaxy types and SFRs in more elongated and less elongated superclusters of spider type are different at very high significance level. Costa-Duarte et al. (2013) did not find differences in galaxy populations in superclusters of different shape, and we found this for spider-type systems only. We analysed quite rich systems only, diving them at first by their inner morphology. This may lead to the differences in our results.

\subsection{The richest superclusters}

Next we compared galaxy populations in the richest individual superclusters of both morphological types, the superclusters of filament morphology, $\mathrm{SCl} 001$ and $\mathrm{SCl} 027$, and the superclusters of spider morphology, SCL 019 and SCl 099. The superclusters SCl 027 and SCl 019 are the richest systems in the Sloan Great Wall (Einasto et al. 2011b). The superclusters SCl 099 (the Corona Borealis supercluster) and $\mathrm{SCl} 001$ belong to the dominant supercluster plane (Einasto et al. 1997, 2011a). Figure 8 shows galaxy populations in these superclusters, and Table 2 the results of the KS test, where we compare galaxy content of filament-type systems and spider-type systems.

The galaxy populations in the individual richest systems are all different. Comparison of the galaxy populations of the richest filament-type superclusters and spider-type superclusters shows that the distributions of all their galaxy properties considered in this paper are different at very high significance level, the fractions of red, low SFR galaxies in the richest filament-type systems are higher than those in the richest spider-type systems. For the richest filament-type systems Table 2 shows that all their galaxy properties except stellar masses are different at very high significance level, the fraction of red galaxies in the $\mathrm{SCl} 001$ is higher than in $\mathrm{SCl}$ 027, and the fraction of galaxies with high SFR is lower. For the richest spider-type superclusters only the stellar masses of galaxies in them are different at statistically high significance level, galaxies in SCl 099 having higher stellar masses than in those $\mathrm{SCl} 019$. 

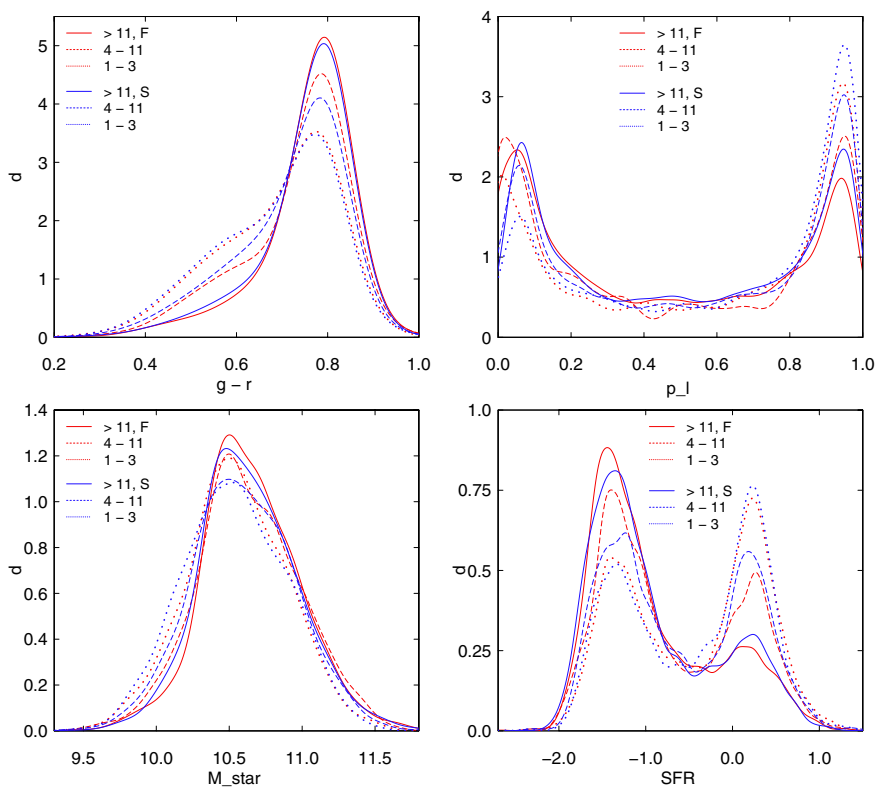

Fig. 9. Same as in Fig. 3 for groups of different richness in superclusters of filament and spider morphology (red and blue lines). Solid lines show distributions for groups with at least 12 member galaxies. Dashed lines correspond to distributions for groups with $4 \leq N_{\text {gal }} \leq 11$. Dotted lines to single galaxies and groups with $N_{\text {gal }} \leq 3$.

\subsection{Rich and poor groups in superclusters}

Most galaxies are located in groups of various richness. Therefore, as a next step we compared the galaxy content of groups of different richness in filament- and spider-type superclusters and in the field. We divided groups of galaxies in superclusters and in the field according to their richness as very poor systems with $N_{\text {gal }} \leq 3$, poor groups with the number of member galaxies $4 \leq N_{\text {gal }} \leq 11$, and rich groups with $N_{\text {gal }} \geq 12$. The comparison of galaxy properties in three richness classes are given in Fig. 9. For clarity we present in Fig. 9 the results for groups from superclusters only. Table C.4 presents the values of quantiles of galaxy properties.

Figure 9 shows that the richer the group, the larger is the fraction of red, early-type, low SFR, high stellar mass galaxies in it. We found a similar trend for groups in low-density global environments. The KS test shows that differences between all galaxy parameters from poorer and richer groups are statistically highly significant.

Figure 9 shows that colour distributions of galaxies in very poor groups with $N_{\text {gal }} \leq 3$ are very similar in superclusters of different type. The KS test shows that the differences are not statistically significant (Table 3 ). Galaxies in very poor groups in superclusters of filament-type have higher stellar masses than galaxies in superclusters of spider-type, and the fraction of high SFR galaxies among them is smaller. For richer groups Fig. 9 shows that groups of the same richness host galaxies with higher stellar masses, and they have a larger fraction of early-type and red galaxies, and a higher fraction of low SFR galaxies, if they are located in superclusters of filament morphology. For colours and SFRs the KS test says that the differences between groups with less and more than 12 galaxies are not statistically significant. The stellar masses and types of galaxies are also significantly different in the case of these richness classes. In the field groups the fraction of red, low SFR galaxies is lower than in groups of the same richness in superclusters. Summarising, the
Table 3. Results of the KS test (the $p_{\mathrm{KS}}$-values): galaxy properties in groups of different richness in superclusters of filament and spider morphology.

\begin{tabular}{lccc}
\hline \hline$(1)$ & $(2)$ & $(3)$ & $(4)$ \\
\hline Property & $N_{\text {gal }}<4$ & $4 \leq N_{\text {gal }} \leq 11$ & $N_{\text {gal }} \geq 12$ \\
& $p_{\text {KS }}$ & $p_{\text {KS }}$ & $p_{\text {KS }}$ \\
\hline$g-r$ & 0.359 & $9 e-05$ & 0.122 \\
$p_{1}$ & $<1 e-5$ & $<1 e-5$ & $<1 e-5$ \\
$\log M_{\mathrm{s}}$ & 0.006 & 0.017 & 0.014 \\
$S F R$ & $3 \mathrm{e}-04$ & 0.022 & 0.388 \\
\hline
\end{tabular}

Notes. Columns are as follows: 1: Galaxy properties are the same as in Table 2. 2: the $p_{\mathrm{KS}}$-values of the test for single galaxies and groups with 2-3 member galaxies in superclusters of filament and spider morphology. 3: the same for groups with 4-11 member galaxies. 4: the same for groups with at least 12 member galaxies.

galaxy content of groups of the same richness is somewhat different in superclusters of filament and spider morphology.

\subsection{The peculiar velocities of the main galaxies of groups}

Einasto et al. (2012a) showed that in superclusters of spider morphology rich groups with $N_{\text {gal }} \geq 50$ have a greater probability of having substructure and higher peculiar velocities $\left|v_{\text {pec }}\right|$ of their main galaxies than rich groups in superclusters of filament morphology. They suggested that rich groups in superclusters of spider morphology may be dynamically younger than rich groups in superclusters of filament morphology. Next we compare the peculiar velocities of the main galaxies of groups in superclusters and in the field to obtain an estimate of the group's dynamical state, and to understand whether the differences in the dynamical state of groups may be related to the differences in their galaxy content. In these calculations we did not want to use data of all groups since for very poor groups calculations of the peculiar velocities of their main galaxies are not reliable. Recently Ribeiro et al. (2013) used several methods to analyse the structure and dynamical state of galaxy groups and showed that these methods give quite reliable results for groups with more than ten member galaxies (Fig. 2 and Table 1 in Ribeiro et al. 2013). We made calculations with several group richness limits and found that the results are similar, but for richer groups the sample size is smaller and this decreases the statistical significance of the results. Therefore we used groups with $N_{\text {gal }} \geq 12$ in our analysis. We show the distributions of the values of the peculiar velocities of the main galaxies in groups in superclusters and in the field in Fig. 10 (left panel).

Figure 10 shows that the distributions are clearly different. The KS test says that the differences of the peculiar velocities of the main galaxies in groups with at least 12 member galaxies in superclusters of filament and spider morphology, and between supercluster and field galaxy groups are statistically significant at very high levels ( $p$-value $p<0.05$ ). There is a deficit of groups with small values of the peculiar velocities of their main galaxies in groups from superclusters of filament morphology, and an excess in groups from superclusters of spider morphology, and in the field. Einasto et al. (2012b) showed for very rich groups with $N_{\text {gal }} \geq 50$ that in groups with the peculiar velocities of the main galaxies $\left|v_{\text {pec }}\right|<250 \mathrm{~km} \mathrm{~s}^{-1}$ main galaxies are located near the centre of the group. Higher peculiar velocities of the main galaxies suggest that the main galaxy is located far from the centre, a sign of a dynamically young group. Figure 10 shows that the peculiar velocities of the main galaxies in groups 

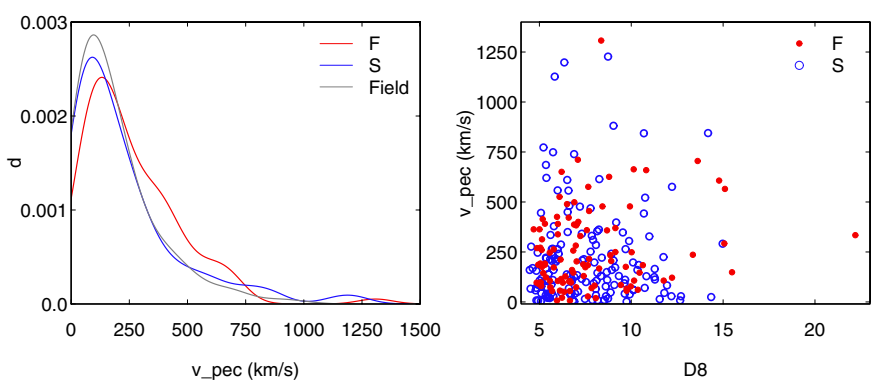

Fig. 10. Left panel: peculiar velocities of the main galaxies in groups with at least 12 member galaxies in superclusters of filament and spider morphology (red and blue lines), and in the field (grey line). Right panel: peculiar velocities of the main galaxies in groups with at least 12 member galaxies in superclusters of filament and spider morphology (red and blue dots) vs. environmental density in superclusters at smoothing length $8 h^{-1}$ Mpc.

in superclusters of filament morphology are higher than those in groups from superclusters of spider morphology, suggesting that they are dynamically younger. In the richest groups we see the opposite: here the peculiar velocities of the main galaxies in groups from spider-type superclusters are higher than those in groups of filament-type superclusters, as also shown in Einasto et al. (2012a). Interestingly, we found that groups of the same richness contain a larger fraction of red, passive galaxies in superclusters of filament morphology than in superclusters of spider morphology, and this trend is similar for groups of different richness, but the values of the peculiar velocities depend on richness.

We also studied the relation between the peculiar velocities of group main galaxies, and environmental density in superclusters around them, for several smoothing lengths. Since the results were similar, we present them for smoothing length $8 h^{-1} \mathrm{Mpc}$ only (Fig. 10, right panel). This figure shows a possible weak correlation between the peculiar velocities of the main galaxies and environmental density for filament-type superclusters, but not for spider-type superclusters. This was confirmed by Pearson's correlation test which showed for filament-type superclusters that the correlation coeficient $r=0.19$ with $p=0.06$. For spider-type superclusters this test found that $r=0.01$ with $p=0.93$.

\section{Discussion and conclusions}

We showed that the fraction of red, early-type, low SFR galaxies in superclusters of filament morphology is higher than in superclusters of spider morphology. In addition, the fraction of these galaxies in superclusters is higher than among galaxies in low-density global environments. There are relatively more red galaxies among low stellar mass galaxies in spider-type superclusters than in filament-type superclusters.

In superclusters of filament morphology groups of equal richness host galaxies with higher stellar masses, and they have a larger fraction of early type and red galaxies, and a higher fraction of low SFR galaxies in comparison with groups in spidertype superclusters. In agreement with Lietzen et al. (2012), we found that groups of equal richness have a larger fraction of early-type and red galaxies, and a higher fraction of low SFR galaxies, if they are located in high-density large-scale environments (superclusters) rather than in the field. In superclusters they also host galaxies with higher stellar masses than groups in the field. Luparello et al. (2013) also found that galaxy groups in superstructures (Luparello et al. 2011) have higher stellar masses, higher values of the peculiar velocities, and have older galaxy populations than groups outside of superstructures.

About $10 \%$ of all galaxies in both types of superclusters are red, high SFR galaxies. These galaxies are mostly late type, their stellar masses are similar to stellar masses of red, low SFR galaxies, and higher than those of blue galaxies. In spidertype superclusters their stellar masses have higher values than in filament-type superclusters. Earlier studies have shown that massive galaxies are red, independent of their morphological types (Bamford et al. 2009; Masters et al. 2010; Einasto et al. 2011b). We found that red, high SFR galaxies can usually be found at low and intermediate densities in both type of superclusters, but some of them are also located at regions of quite high environmental density. Red, high SFR galaxies typically lie at intermediate densities in small groups in superclusters (Wolf et al. 2005; Einasto et al. 2008; Skibba et al. 2009), but they can be also found in rich clusters, and among the main galaxies of clusters (Einasto et al. 2011b), in agreement with our present findings. This means that processes which change the colours of late-type galaxies to red while keeping the high SFR must occur both in high- and intermediate-density regions, and are more effective on high stellar mass galaxies. We plan to study their location in groups and clusters of individual superclusters to understand their distribution and properties better.

Einasto et al. (2008, 2011b) determined a large variation in the distributions of different galaxy populations in individual rich superclusters. We found in the present study a large diversity in the properties of galaxy populations of the richest superclusters in our sample. Explaining large variation in the distribution of galaxy populations in superclusters is a challenge for galaxy formation models.

Costa-Duarte et al. (2013) showed that there is no difference between stellar populations of galaxies in superclusters classified by their overall shape as filaments and pancakes. In our study we found that when we divided superclusters of different morphology by their shape parameter into more elongated and less elongated populations then the differences in galaxy population between them are small.

Superclusters obtained their morphology and group and galaxy populations during the formation and evolution of the cosmic web. In the $\Lambda$ Cold Dark Matter $(\Lambda C D M)$ concordance cosmological model the structures forming the cosmic web grow by hierarchical clustering driven by gravity (see Loeb 2002, 2008, and references therein). The present-day dynamical state of clusters of galaxies depends on their formation history. We analysed the dynamical state of galaxy groups using the peculiar velocity of their main galaxies as an indicator of the dynamical state, and found that the velocities in filament-type superclusters are higher than those in spider-type superclusters. In this analysis we used groups with $N_{\text {gal }} \geq 12$. In contrast, for the richest clusters with $N_{\text {gal }} \geq 50$ Einasto et al. (2012a) found that these velocities are higher in superclusters of spider morphology.

Krause et al. (2013) recently analysed the distribution and dynamical state of galaxy groups in the Ursa Major Supercluster (in our catalogue SCl 211 of filament morphology) and found that groups with Gaussian velocity distribution of their member galaxies are located in the denser regions of the supercluster. They suggested that relaxed galaxy groups in the supercluster have formed and evolved earlier and faster around highdensity peaks, while nonrelaxed systems may be growing more slowly on the peripheries of lower density peaks. Interestingly, we found in this study a weak correlation between environmental density and the peculiar velocities of the main galaxies in 
groups in filament-type superclusters, so that groups with higher values of the peculiar velocities of their main galaxies are located in higher density environments, this is opposite to the Krause et al. (2013) result. They studied one rich supercluster. We showed that the properties of individual rich superclusters vary strongly. This may be the reason for different results. Einasto et al. (2011b) found in the study of the richest superclusters in the Sloan Great Wall, SCl 027 and SCl 019, that the peculiar velocities of the main galaxies in groups depend on the morphology of the main galaxies, and on their location in the supercluster.

Einasto et al. (2012a) assumed that superclusters of spider morphology have richer inner structure than superclusters of filament morphology with a large number of filaments between clusters in them so mergers of clusters may occur more often in these superclusters. Therefore clusters in spider-type superclusters could be dynamically younger. This agrees with the results of their galaxy populations, spider-type superclusters hosting a larger fraction of blue, star forming galaxies, but contradicts the calculations of the peculiar velocities of their main galaxies. This is one of the open questions for future studies of superclusters and galaxy clusters in them.

Einasto et al. (2005) showed by numerical simulations that in low-density global environment only poor systems form, these systems grow very slowly. In high-density global environments, the dynamical evolution is rapid and starts earlier, consisting of a continua of transitions of dark matter particles to building blocks of galaxies, groups, and clusters. Dark matter halos in voids are found to be less massive, less luminous, and their growth of mass is suppressed and stops at earlier epochs than in high-density regions. During the structure formation in the Universe, halo sizes in the supercluster core regions increase, while in void regions halo sizes remain unchanged (Tempel et al. 2009). Thus, during the evolution of structure the overall density in superclusters increases, which enhances the evolution of the small-scale protohalos in them (Suhhonenko et al. 2011). This may lead to the differences in the properties of galaxy groups and galaxies in them in superclusters and in the field.

A number of processes that transform galaxies in highdensity environments have been proposed, either observationally or in simulations. These include galaxy-galaxy mergers (Barnes \& Hernquist 1992), galaxy harassment (Richstone 1976; Moore et al. 1996), tidal stripping by the host group or cluster's gravitational potential (Gnedin 2003), ram pressure stripping of cold gas by the hot gas of the diffuse intra-cluster or intra-group medium (Gunn \& Gott 1972), and strangulation or removal of galactic halo gas (Larson et al. 1980). These processes are effective both in galaxy groups and during mergers of groups into clusters (pre- and post-processing of galaxies, Berrier et al. 2009; Vijayaraghavan \& Ricker 2013).

Cen (2011) used hydrodynamical simulations to show that for galaxies at $z=0$ star formation is efficient in low-density regions while it is substantially suppressed in cluster environments. During hierarchical structure formation, gas is heated in high-density regions (groups, clusters, and superclusters), and an increasingly larger fraction of the gas has entered a phase that is too hot to feed the residing galaxies. As a result, the cold gas supply to galaxies in these regions is suppressed. The net effect is that star formation gradually shifts from the larger halos that populate overdense regions to lower density environments. Thus, a lack of cold gas due to gravitational heating in dense regions may provide a physical explanation of cosmic downsizing and one of its manifestations is observed as the colour-density relation.
Summarising, our results are as follows.

1) The fraction of red, early-type, low SFR galaxies is higher in superclusters of filament-type than in superclusters of spidertype, higher in superclusters than among galaxies in lowdensity global environment, and higher in all environments in rich groups than in poor groups.

2) Groups of equal richness host galaxies with higher stellar masses, and they have a larger fraction of early-type and red galaxies, and a higher fraction of low SFR galaxies if they are located in superclusters of filament morphology.

3) In spider-type superclusters the red, high SFR galaxies have higher stellar masses and lower values of environmental densities around them than in filament-type superclusters.

4) The peculiar velocities of the main galaxies in groups in superclusters of filament morphology are higher than in groups in superclusters of spider morphology. In filament-type superclusters groups with higher peculiar velocities of their main galaxies are located in higher density environments than those with low peculiar velocities.

5) There are significant differences between galaxy populations of the individual richest superclusters.

These results suggest that both local (group) and global (supercluster) environments, and inner structure and detailed density distribution in superclusters are important factors affecting the evolution of galaxies and galaxy groups in them. Our study indicated several open questions that need future studies. It is not clear how the processes that shape the properties of galaxies in different environments are related to the supercluster scale density field, and how the supercluster morphology is related to the properties of galaxies and groups in them. We plan to develop further the methods to quantify inner structure of superclusters, in order to understand better the role of supercluster environment in the formation and evolution of galaxies and galaxy groups in them. We also plan to study the inner structure of superclusters using galactic filaments (Tempel et al. 2014).

Acknowledgements. We thank the referee for invaluable comments and suggestions which helped to improve the paper. We thank Enn Saar for very fruitful collaboration on studies of supercluster morphology. We are pleased to thank the SDSS Team for the publicly available data releases. Funding for the Sloan Digital Sky Survey (SDSS) and SDSS-II has been provided by the Alfred P. Sloan Foundation, the Participating Institutions, the National Science Foundation, the US Department of Energy, the National Aeronautics and Space Administration, the Japanese Monbukagakusho, and the Max Planck Society, and the Higher Education Funding Council for England. The SDSS Web site is http://www . sdss.org/. The SDSS is managed by the Astrophysical Research Consortium (ARC) for the Participating Institutions. The Participating Institutions are the American Museum of Natural History, Astrophysical Institute Potsdam, University of Basel, University of Cambridge, Case Western Reserve University, The University of Chicago, Drexel University, Fermilab, the Institute for Advanced Study, the Japan Participation Group, The Johns Hopkins University, the Joint Institute for Nuclear Astrophysics, the Kavli Institute for Particle Astrophysics and Cosmology, the Korean Scientist Group, the Chinese Academy of Sciences (LAMOST), Los Alamos National Laboratory, the Max-PlanckInstitute for Astronomy (MPIA), the Max-Planck-Institute for Astrophysics (MPA), New Mexico State University, Ohio State University, University of Pittsburgh, University of Portsmouth, Princeton University, the United States Naval Observatory, and the University of Washington. The present study was supported by the Estonian Science Foundation grant No. 8005, grants 9428, MJD272, PUT246, by the Estonian Ministry for Education and Science research project SF0060067s08, and by the European Structural Funds grant for the Centre of Excellence "Dark Matter in (Astro)particle Physics and Cosmology" TK120. This work has also been supported by ICRAnet through a professorship for Jaan Einasto. H. Lietzen acknowledges financial support from the Spanish Ministry of Economy and Competitiveness (MINECO) under the 2011 Severo Ochoa Program MINECO SEV-2011-0187. 


\section{References}

Aihara, H., Allende Prieto, C., An, D., et al. 2011, ApJS, 193, 29

Bamford, S. P., Nichol, R. C., Baldry, I. K., et al. 2009, MNRAS, 393, 1324

Barnes, J. E., \& Hernquist, L. 1992, ARA\&A, 30, 705

Berrier, J. C., Stewart, K. R., Bullock, J. S., et al. 2009, ApJ, 690, 1292

Blanton, M. R., \& Moustakas, J. 2009, ARA\&A, 47, 159

Blanton, M. R., \& Roweis, S. 2007, AJ, 133, 734

Blanton, M. R., Hogg, D. W., Bahcall, N. A., et al. 2003, ApJ, 592, 819

Brinchmann, J., Charlot, S., White, S. D. M., et al. 2004, MNRAS, 351, 1151

Bruzual, G., \& Charlot, S. 2003, MNRAS, 344, 1000

Cen, R. 2011, ApJ, 741, 99

Costa-Duarte, M. V., Sodré, Jr., L., \& Durret, F. 2011, MNRAS, 411, 1716

Costa-Duarte, M. V., Sodré, L., \& Durret, F. 2013, MNRAS, 428, 906

Coziol, R., Andernach, H., Caretta, C. A., Alamo-Martínez, K. A., \& Tago, E. 2009, AJ, 137, 4795

de Vaucouleurs, G. 1956, Vistas in Astron., 2, 1584

Dressler, A. 1980, ApJ, 236, 351

Einasto, J., Saar, E., Kaasik, A., \& Chernin, A. D. 1974, Nature, 252, 111

Einasto, J., Klypin, A. A., Saar, E., \& Shandarin, S. F. 1984, MNRAS, 206, 529

Einasto, J., Tago, E., Einasto, M., et al. 2005, A\&A, 439, 45

Einasto, J., Einasto, M., Saar, E., et al. 2007, A\&A, 462, 397

Einasto, M. 1991, MNRAS, 252, 261

Einasto, M., \& Einasto, J. 1987, MNRAS, 226, 543

Einasto, M., Einasto, J., Tago, E., Dalton, G. B., \& Andernach, H. 1994, MNRAS, 269, 301

Einasto, M., Tago, E., Jaaniste, J., Einasto, J., \& Andernach, H. 1997, A\&AS, 123, 119

Einasto, M., Einasto, J., Tago, E., Müller, V., \& Andernach, H. 2001, AJ, 122, 2222

Einasto, M., Einasto, J., Müller, V., Heinämäki, P., \& Tucker, D. L. 2003a, A\&A, 401,851

Einasto, M., Jaaniste, J., Einasto, J., et al. 2003b, A\&A, 405, 821

Einasto, M., Einasto, J., Tago, E., et al. 2007a, A\&A, 464, 815

Einasto, M., Saar, E., Liivamägi, L. J., et al. 2007b, A\&A, 476, 697

Einasto, M., Saar, E., Martínez, V. J., et al. 2008, ApJ, 685, 83

Einasto, M., Liivamägi, L. J., Tago, E., et al. 2011a, A\&A, 532, A5

Einasto, M., Liivamägi, L. J., Tempel, E., et al. 2011b, ApJ, 736, 51

Einasto, M., Liivamägi, L. J., Tempel, E., et al. 2012a, A\&A, 542, A36

Einasto, M., Vennik, J., Nurmi, P., et al. 2012b, A\&A, 540, A123

Gnedin, O. Y. 2003, ApJ, 582, 141

Gunn, J. E., \& Gott, III, J. R. 1972, ApJ, 176, 1

Hamilton, A. J. S. 1988, ApJ, 331, L59

Huchra, J. P., \& Geller, M. J. 1982, ApJ, 257, 423

Huertas-Company, M., Aguerri, J. A. L., Bernardi, M., Mei, S., \& Sánchez Almeida, J. 2011, A\&A, 525, A157

Ihaka, R., \& Gentleman, R. 1996, J. Comput. Graph. Stat., 5, 299
Jõeveer, M., Einasto, J., \& Tago, E. 1978, MNRAS, 185, 357

Kauffmann, G., Heckman, T. M., White, S. D. M., et al. 2003, MNRAS, 341, 33 Kirshner, R. P., Oemler, Jr., A., Schechter, P. L., \& Shectman, S. A. 1981, ApJ, 248, L57

Komatsu, E., Smith, K. M., Dunkley, J., et al. 2011, ApJS, 192, 18

Krause, M. O., Ribeiro, A. L. B., \& Lopes, P. A. A. 2013, A\&A, 551, A143

Larson, R. B., Tinsley, B. M., \& Caldwell, C. N. 1980, ApJ, 237, 692

Lietzen, H., Tempel, E., Heinämäki, P., et al. 2012, A\&A, 545, A104

Liivamägi, L. J., Tempel, E., \& Saar, E. 2012, A\&A, 539, A80

Loeb, A. 2002, Phys. Rev. D, 65, 047301

Loeb, A. 2008 [arXiv: 0804.2258]

Luparello, H., Lares, M., Lambas, D. G., \& Padilla, N. 2011, MNRAS, 415, 964

Luparello, H. E., Lares, M., Yaryura, C. Y., et al. 2013, MNRAS, 432, 1367

Martínez, V. J., \& Saar, E. 2002, Statistics of the Galaxy Distribution (Boca Raton: Chapman \& Hall/CRC)

Masters, K. L., Mosleh, M., Romer, A. K., et al. 2010, MNRAS, 405, 783

Mo, H. J., Einasto, M., Xia, X. Y., \& Deng, Z. G. 1992, MNRAS, 255, 382

Moore, B., Katz, N., Lake, G., Dressler, A., \& Oemler, A. 1996, Nature, 379, 613

Ostriker, J. P., \& Tremaine, S. D. 1975, ApJ, 202, L113

Park, C., Choi, Y., Vogeley, M. S., Gott, III, J. R., \& Blanton, M. R. 2007, ApJ, 658, 898

Postman, M., \& Geller, M. J. 1984, ApJ, 281, 95

Ribeiro, A. L. B., de Carvalho, R. R., Trevisan, M., et al. 2013, MNRAS, 434, 784

Richstone, D. O. 1976, ApJ, 204, 642

Saar, E. 2009, in Data Analysis in Cosmology, eds. V. J. Martínez, E. Saar, E. Martínez-Gonzalez, \& M.-J. Pons-Bordería (Berlin: Springer-Verlag), 523

Saar, E., Martínez, V. J., Starck, J., \& Donoho, D. L. 2007, MNRAS, 374, 1030 Sahni, V., Sathyaprakash, B. S., \& Shandarin, S. F. 1998, ApJ, 495, L5

Salim, S., Rich, R. M., Charlot, S., et al. 2007, ApJS, 173, 267

Shandarin, S. F., Sheth, J. V., \& Sahni, V. 2004, MNRAS, 353, 162

Skibba, R. A. 2009, MNRAS, 392, 1467

Skibba, R. A., Bamford, S. P., Nichol, R. C., et al. 2009, MNRAS, 399, 966

Suhhonenko, I., Einasto, J., Liivamägi, L. J., et al. 2011, A\&A, 531, A149

Tago, E., Einasto, J., Saar, E., et al. 2008, A\&A, 479, 927

Tago, E., Saar, E., Tempel, E., et al. 2010, A\&A, 514, A102

Tempel, E., Einasto, J., Einasto, M., Saar, E., \& Tago, E. 2009, A\&A, 495, 37

Tempel, E., Saar, E., Liivamägi, L. J., et al. 2011, A\&A, 529, A53

Tempel, E., Tago, E., \& Liivamägi, L. J. 2012, A\&A, 540, A106

Tempel, E., Stoica, R. S., Martínez, V. J., et al. 2014, MNRAS, in press [arXiv: 1308.2533]

Tremonti, C. A., Heckman, T. M., Kauffmann, G., et al. 2004, ApJ, 613, 898

Vijayaraghavan, R., \& Ricker, P. M. 2013, MNRAS, 435, 2713

Wolf, C., Gray, M. E., \& Meisenheimer, K. 2005, A\&A, 443, 435

Zeldovich, I. B., Einasto, J., \& Shandarin, S. F. 1982, Nature, 300, 407

Zucca, E., Zamorani, G., Scaramella, R., \& Vettolani, G. 1993, ApJ, 407, 470

Pages 11 to 14 are available in the electronic edition of the journal at http://www. aanda.org 


\section{Appendix A: Supercluster data}

In Table A. 1 we present data on superclusters.

Table A.1. Supercluster data.

\begin{tabular}{|c|c|c|c|c|c|c|c|c|}
\hline (1) & (2) & (3) & (4) & (5) & $(6)$ & (7) & (8) & (9) \\
\hline ID(long) & ID & $N_{\text {gal }}$ & $\begin{array}{c}d \\
h^{-1} \mathrm{Mpc}\end{array}$ & $\begin{array}{c}L_{\mathrm{tot}} \\
10^{10} h^{-2} L_{\odot}\end{array}$ & $V_{3}$ & $K_{1} / K_{2}$ & Type & $\mathrm{ID}(\mathrm{E} 01)$ \\
\hline $239+027+0091$ & 1 & 1041 & 264 & 1809 & 2.5 & 0.41 & $\mathrm{~F}$ & 162 \\
\hline $227+006+0078$ & 7 & 1217 & 233 & 1675 & 3.0 & 0.56 & $\mathrm{~S}$ & 154 \\
\hline $184+003+0077$ & 19 & 2060 & 231 & 2919 & 9.0 & 0.33 & $\mathrm{~S}$ & 111 \\
\hline $167+040+0078$ & 24 & 580 & 225 & 751 & 2.0 & 0.86 & $\mathrm{~S}$ & 95 \\
\hline $202-001+0084$ & 27 & 3222 & 256 & 5163 & 14.5 & 0.26 & $\mathrm{~F}$ & 126 \\
\hline $173+014+0082$ & 54 & 1341 & 241 & 2064 & 5.5 & 0.56 & $\mathrm{~S}$ & 111 \\
\hline $189+017+0071$ & 89 & 515 & 212 & 610 & 2.0 & 1.98 & $\mathrm{~S}$ & 271 \\
\hline $215+048+0071$ & 92 & 527 & 213 & 690 & 2.5 & 0.40 & $\mathrm{~S}$ & - \\
\hline $230+027+0070$ & 99 & 2047 & 215 & 2874 & 10.0 & 0.26 & $\mathrm{~S}$ & 158 \\
\hline $203+059+0072$ & 143 & 668 & 211 & 753 & 2.0 & 1.27 & $\mathrm{~F}$ & 133 \\
\hline $172+054+0071$ & 211 & 1439 & 207 & 1618 & 7.0 & 0.36 & $\mathrm{~F}$ & 109 \\
\hline $187+008+0090$ & 214 & 735 & 268 & 1218 & 6.0 & 0.28 & $\mathrm{~S}$ & 111 \\
\hline $197+039+0073$ & 218 & 272 & 215 & 337 & 1.0 & -20.02 & $\mathrm{~S}$ & 274 \\
\hline $207+026+0067$ & 219 & 985 & 187 & 1007 & 4.0 & 0.61 & $\mathrm{~S}$ & 138 \\
\hline $207+028+0077$ & 220 & 603 & 226 & 768 & 4.0 & 0.47 & $\mathrm{~F}$ & 138 \\
\hline $255+033+0086$ & 233 & 474 & 259 & 790 & 4.0 & 0.96 & $\mathrm{~F}$ & 167 \\
\hline $122+035+0084$ & 298 & 246 & 246 & 345 & 1.0 & 4.27 & $\mathrm{~S}$ & 75 \\
\hline $159+004+0069$ & 319 & 245 & 207 & 296 & 2.0 & 0.97 & $\mathrm{~S}$ & 91 \\
\hline $195+019+0064$ & 344 & 264 & 192 & 290 & 1.0 & -0.14 & $\mathrm{~S}$ & 273 \\
\hline $216+016+0051$ & 349 & 335 & 159 & 284 & 1.0 & 0.97 & $\mathrm{~S}$ & 143 \\
\hline $227+007+0045$ & 352 & 519 & 135 & 379 & 1.0 & 1.46 & $\mathrm{~S}$ & 154 \\
\hline $232+029+0066$ & 360 & 311 & 196 & 330 & 2.0 & -0.54 & $\mathrm{~S}$ & 158 \\
\hline $146+043+0072$ & 491 & 199 & 217 & 256 & 1.0 & -0.15 & $\mathrm{~S}$ & - \\
\hline $168+002+0077$ & 499 & 408 & 228 & 517 & 3.0 & 0.67 & $\mathrm{~F}$ & 91 \\
\hline $176+055+0052$ & 515 & 457 & 155 & 390 & 3.0 & -11.10 & S & 109 \\
\hline $208+046+0062$ & 532 & 297 & 189 & 293 & 2.0 & 0.49 & $\mathrm{~F}$ & - \\
\hline $214+002+0053$ & 540 & 422 & 163 & 358 & 1.5 & 0.49 & $\mathrm{~S}$ & - \\
\hline $223+016+0045$ & 541 & 299 & 135 & 214 & 1.0 & -0.88 & S & - \\
\hline $129+028+0079$ & 721 & 94 & 237 & 145 & 1.0 & 0.22 & $\mathrm{~S}$ & 76 \\
\hline $151+054+0047$ & 782 & 652 & 139 & 465 & 3.0 & 0.38 & $\mathrm{~S}$ & - \\
\hline $169+029+0046$ & 793 & 211 & 142 & 156 & 1.0 & -1.24 & S & 93 \\
\hline $176+015+0069$ & 797 & 160 & 205 & 203 & 1.0 & 0.22 & $\mathrm{~S}$ & - \\
\hline $240+053+0065$ & 849 & 215 & 194 & 242 & 1.0 & -0.03 & $\mathrm{~F}$ & 162 \\
\hline $244+049+0057$ & 850 & 135 & 171 & 128 & 1.0 & 0.63 & $\mathrm{~S}$ & 162 \\
\hline $234+036+0065$ & 865 & 161 & 197 & 170 & 2.0 & -1.68 & S & 158 \\
\hline $246+014+0050$ & 868 & 239 & 153 & 201 & 1.5 & 0.98 & $\mathrm{~S}$ & - \\
\hline $219+024+0087$ & 870 & 76 & 261 & 143 & 1.0 & -0.14 & $\mathrm{~S}$ & - \\
\hline $127+030+0051$ & 1104 & 127 & 150 & 110 & 1.0 & 0.68 & S & - \\
\hline $191+054+0085$ & 1192 & 79 & 248 & 134 & 1.0 & 1.41 & S & - \\
\hline $220+010+0051$ & 1238 & 133 & 156 & 97 & 1.0 & -0.28 & $\mathrm{~F}$ & - \\
\hline $226+028+0057$ & 1244 & 101 & 174 & 97 & 1.0 & 0.76 & $\mathrm{~S}$ & 152 \\
\hline $223+018+0059$ & 1247 & 147 & 176 & 149 & 1.0 & 0.28 & $\mathrm{~F}$ & - \\
\hline
\end{tabular}

Notes. The columns are: 1: ID of the supercluster AAA+BBB+ZZZZ, where AAA is RA, +/-BBB is Dec. (in degrees), and ZZZZ is 1000z; 2: ID of the supercluster from the SDSS DR8 superclusters catalogue (Einasto et al. 2012a); 3: the number of galaxies in the supercluster, $N_{\text {gal }}$; 4: the distance of the supercluster; 5 : the total weighted luminosity of galaxies in the supercluster, $L_{\text {tot }}$; 6 : the maximum value of the fourth Minkowski functional, $V_{3, \max }$ (clumpiness), for the supercluster; 7 : the ratio of the shapefinders $K_{1} / K_{2}$ (the shape parameter) for the supercluster; 8: Morphological type of a supercluster; 9: ID(E01): the supercluster ID in the catalogue by Einasto et al. (2001). SCl 111 and SCl 126 - members of the Sloan Great Wall, SCl 158 - the Corona Borealis supercluster, SCl 138 - the Bootes supercluster, SCl 109 - the Ursa Major supercluster. 


\section{Appendix B: Minkowski functionals and shapefinders}

For a given surface the four Minkowski functionals (from the first to the fourth) are proportional to the enclosed volume $V$, the area of the surface $S$, the integrated mean curvature $C$, and the integrated Gaussian curvature $\chi$. Consider an excursion set $F_{\phi_{0}}$ of a field $\phi(x)$ (the set of all points where the density is higher than a given limit, $\left.\phi(\boldsymbol{x}) \geq \phi_{0}\right)$. Then, the first Minkowski functional is the volume of this region (the excursion set):

$V_{0}\left(\phi_{0}\right)=\int_{F_{\phi_{0}}} \mathrm{~d}^{3} x$

The second Minkowski functional is proportional to the surface area of the boundary $\delta F_{\phi}$ of the excursion set:

$V_{1}\left(\phi_{0}\right)=\frac{1}{6} \int_{\delta F_{\phi_{0}}} \mathrm{~d} S(\boldsymbol{x})$.

The third Minkowski functional is proportional to the integrated mean curvature $C$ of the boundary:

$V_{2}\left(\phi_{0}\right)=\frac{1}{6 \pi} \int_{\delta F_{\phi_{0}}}\left(\frac{1}{R_{1}(\boldsymbol{x})}+\frac{1}{R_{2}(\boldsymbol{x})}\right) \mathrm{d} S(\boldsymbol{x})$,

where $R_{1}(\boldsymbol{x})$ and $R_{2}(\boldsymbol{x})$ are the principal radii of curvature of the boundary. The fourth Minkowski functional is proportional to the integrated Gaussian curvature (the Euler characteristic) of the boundary:

$V_{3}\left(\phi_{0}\right)=\frac{1}{4 \pi} \int_{\delta F_{\phi_{0}}} \frac{1}{R_{1}(\boldsymbol{x}) R_{2}(\boldsymbol{x})} \mathrm{d} S(\boldsymbol{x})$.

At high (low) densities this functional gives us the number of isolated clumps (void cavities) in the sample (Martínez \& Saar 2002; Saar et al. 2007):

$V_{3}=N_{\text {clumps }}+N_{\text {cavities }}-N_{\text {tunnels }}$.

The first three functionals were used to calculate the shapefinders $K_{1}$ (planarity) and $K_{2}$ (filamentarity) (Sahni et al. 1998; Shandarin et al. 2004; Saar 2009) and their ratio, the shape parameter $K_{1} / K_{2}$. The smaller the shape parameter, the more elongated a supercluster is. We used morphological information about superclusters, and their visual appearance to classify them
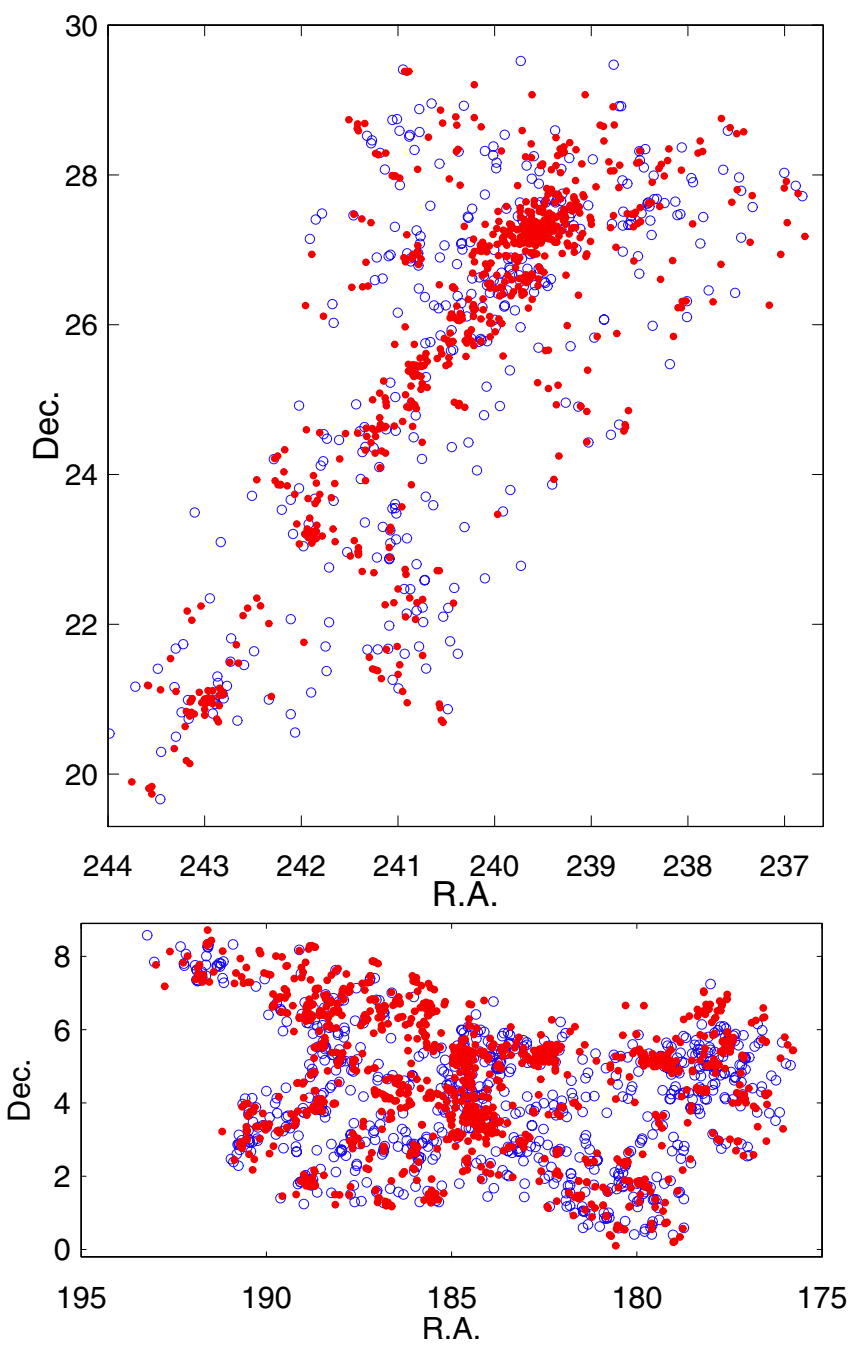

Fig. B.1. Sky distribution of galaxies for the supercluster SCl 001 of filament morphology (upper panel), and for the supercluster SCl 019 of spider morphology (lower panel). Red dots correspond to galaxies with low SFR, $\log S F R<-0.5$, and blue dots to galaxies with high SFR, $\log S F R \geq-0.5$.

as filaments and spiders. As an example, we present in Fig. B.1 the sky distribution of galaxies in one filament-type and one spider-type supercluster (SCL 001 and SCL 019, respectively). 
M. Einasto et al.: SDSS superclusters: morphology and galaxy content

\section{Appendix C: Quantiles of galaxy parameters in different samples.}

Table C.1. Quantiles of galaxy properties in superclusters of filament and spider morphology, and in the field.

\begin{tabular}{|c|c|c|c|}
\hline $\begin{array}{l}\text { (1) } \\
\text { Sample }\end{array}$ & $\begin{array}{l}\text { (2) } \\
1 \text { st } Q\end{array}$ & $\begin{array}{c}(3) \\
\text { Median }\end{array}$ & $\begin{array}{c}(4) \\
3 \mathrm{rd} Q\end{array}$ \\
\hline Filaments & 0.670 & $\begin{array}{c}g-r \\
0.769\end{array}$ & 0.805 \\
\hline Spiders & 0.662 & 0.764 & 0.803 \\
\hline Field & 0.591 & 0.722 & 0.787 \\
\hline Filaments & 0.088 & $\begin{array}{c}p_{1} \\
0.500\end{array}$ & 0.897 \\
\hline Spiders & 0.147 & 0.647 & 0.915 \\
\hline Field & 0.196 & $\begin{array}{c}0.763 \\
\log S F R\end{array}$ & 0.931 \\
\hline Filaments & -1.377 & -0.932 & 0.066 \\
\hline Spiders & -1.368 & -0.909 & 0.083 \\
\hline Field & -1.207 & $\begin{array}{l}-0.262 \\
\log M_{\mathrm{s}}\end{array}$ & 0.249 \\
\hline Filaments & 10.390 & 10.590 & 10.840 \\
\hline Spiders & 10.370 & 10.580 & 10.830 \\
\hline Field & 10.300 & 10.530 & 10.770 \\
\hline
\end{tabular}

Notes. Columns are as follows: 1: sample. 2-4: values of quantiles of galaxy properties (1st quantile, median, and 3rd quantile).

Table C.2. Quantiles of the properties of high and low stellar mass galaxies in superclusters of filament and spider morphology, and in the field.

\begin{tabular}{|c|c|c|c|}
\hline (1) & (2) & (3) & (4) \\
\hline Sample & 1 st $Q$ & Median & $3 \mathrm{rd} Q$ \\
\hline $\log M_{\mathrm{s}}<10.25$ & & $g-r$ & \\
\hline Filaments & 0.459 & 0.528 & 0.606 \\
\hline Spiders & 0.465 & 0.534 & 0.627 \\
\hline Field & 0.451 & 0.512 & 0.575 \\
\hline $\log M_{\mathrm{s}} \geq 10.25$ & & $g-r$ & \\
\hline Filaments & 0.723 & 0.778 & 0.809 \\
\hline Spiders & 0.715 & 0.776 & 0.807 \\
\hline Field & 0.668 & 0.755 & 0.797 \\
\hline $\log M_{\mathrm{S}}<10.25$ & & $p_{1}$ & \\
\hline Filaments & 0.714 & 0.889 & 0.938 \\
\hline Spiders & 0.770 & 0.898 & 0.946 \\
\hline Field & 0.806 & 0.905 & 0.947 \\
\hline $\log M_{\mathrm{s}} \geq 10.25$ & & $p_{1}$ & \\
\hline Filaments & 0.077 & 0.393 & 0.866 \\
\hline Spiders & 0.117 & 0.514 & 0.896 \\
\hline Field & 0.136 & 0.618 & 0.919 \\
\hline $\log M_{\mathrm{S}}<10.25$ & & $\log S F R$ & \\
\hline Filaments & -0.003 & 0.190 & 0.321 \\
\hline Spiders & -0.017 & 0.174 & 0.316 \\
\hline Field & 0.045 & 0.197 & 0.330 \\
\hline $\log M_{\mathrm{s}} \geq 10.25$ & & $\log S F R$ & \\
\hline Filaments & -1.418 & -1.076 & -0.191 \\
\hline Spiders & -1.422 & -1.064 & -0.171 \\
\hline Field & -1.303 & -0.742 & 0.165 \\
\hline
\end{tabular}

Notes. Columns are as follows: 1: sample. 2-4: values of quantiles of galaxy properties (1st quantile, median, and 3rd quantile).
Table C.3. Quantiles of the properties of red, low SFR (1, see Notes), red, high SFR (2), and blue, high SFR (3) galaxies in superclusters of filament and spider morphology.

\begin{tabular}{lcccc}
\hline \hline$(1)$ & $(2)$ & $(3)$ & $(4)$ & $(5)$ \\
Sample & Subsample & 1 st $Q$ & Median & $3 \mathrm{rd} Q$ \\
\hline \multirow{2}{*}{ Filaments } & 1 & 6.055 & 7.376 & 9.518 \\
& 2 & 5.619 & 6.702 & 8.214 \\
& 3 & 5.526 & 6.528 & 7.988 \\
Spiders & 1 & 5.772 & 7.316 & 9.381 \\
& 2 & 5.576 & 6.618 & 8.324 \\
& 3 & 5.488 & 6.514 & 8.392 \\
Filaments & 1 & 0.062 & 0.213 & 0.648 \\
& 2 & 0.232 & 0.807 & 0.951 \\
& 3 & 0.684 & 0.896 & 0.945 \\
Spiders & 1 & 0.078 & 0.264 & 0.714 \\
& 2 & 0.448 & 0.875 & 0.964 \\
& 3 & 0.808 & 0.914 & 0.952 \\
Filaments & 1 & 10.50 & 10.68 & 10.91 \\
& 2 & 10.53 & 10.70 & 10.92 \\
Spiders & 3 & 10.12 & 10.31 & 10.53 \\
& 1 & 10.47 & 10.67 & 10.89 \\
& 2 & 10.54 & 10.74 & 10.95 \\
& 3 & 10.10 & 10.30 & 10.52 \\
\hline
\end{tabular}

Notes. Columns are as follows: 1: supercluster sample. 2: Galaxy populations divided by colour and SFR. 1: red, low SFR galaxies with $g-r \geq 0.7$, and $\log S F R<-0.5 ; 2$ : red, high SFR galaxies with $g-r \geq 0.7$, and $\log S F R \geq-0.5$ and 3: blue, high SFR galaxies with $g-r<0.7$, and $\log S F R \geq-0.5$. 3-5: Values of quantiles of galaxy properties (1st quantile, median, and 3rd quantile). 
Table C.4. Quantiles of the properties of galaxies in groups of different richness in superclusters of filament and spider morphology.

\begin{tabular}{|c|c|c|c|c|}
\hline $\begin{array}{l}\text { (1) } \\
\text { Sample }\end{array}$ & $\begin{array}{c}\text { (2) } \\
\text { Subsample }\end{array}$ & $\begin{array}{l}\text { (3) } \\
1 \mathrm{st} Q\end{array}$ & $\begin{array}{c}\text { (4) } \\
\text { Median }\end{array}$ & $\begin{array}{c}(5) \\
\operatorname{3rd} Q\end{array}$ \\
\hline Filaments & $\begin{array}{c}1-3 \\
4-11 \\
\geq 12\end{array}$ & $\begin{array}{l}10.33 \\
10.41 \\
10.43\end{array}$ & $\begin{array}{c}\log M_{\mathrm{s}} \\
10.54 \\
10.61 \\
10.63\end{array}$ & $\begin{array}{l}10.79 \\
10.86 \\
10.87\end{array}$ \\
\hline Spiders & $\begin{array}{c}1-3 \\
4-11 \\
\geq 12\end{array}$ & $\begin{array}{l}10.29 \\
10.36 \\
10.41\end{array}$ & $\begin{array}{l}10.53 \\
10.58 \\
10.61 \\
S F R\end{array}$ & $\begin{array}{l}10.79 \\
10.84 \\
10.85\end{array}$ \\
\hline Filaments & $\begin{array}{c}1-3 \\
4-11 \\
\geq 12\end{array}$ & $\begin{array}{l}-1.219 \\
-1.355 \\
-1.462\end{array}$ & $\begin{array}{l}-0.296 \\
-0.927 \\
-1.149\end{array}$ & $\begin{array}{c}0.246 \\
0.075 \\
-0.428\end{array}$ \\
\hline Spiders & $\begin{array}{l}1-3 \\
4-11 \\
\geq 12\end{array}$ & $\begin{array}{l}-1.219 \\
-1.309 \\
-1.458\end{array}$ & $\begin{array}{l}-0.276 \\
-0.767 \\
-1.142\end{array}$ & $\begin{array}{r}0.236 \\
0.136 \\
-0.348\end{array}$ \\
\hline Filaments & $\begin{array}{l}1-3 \\
4-11 \\
\geq 12\end{array}$ & $\begin{array}{l}0.605 \\
0.670 \\
0.734\end{array}$ & $\begin{array}{l}g-r \\
0.727 \\
0.771 \\
0.784\end{array}$ & $\begin{array}{l}0.790 \\
0.803 \\
0.813\end{array}$ \\
\hline Spiders & $\begin{array}{c}1-3 \\
4-11 \\
\geq 12\end{array}$ & $\begin{array}{l}0.595 \\
0.646 \\
0.726\end{array}$ & $\begin{array}{l}0.722 \\
0.758 \\
0.781\end{array}$ & $\begin{array}{l}0.786 \\
0.799 \\
0.811\end{array}$ \\
\hline Filaments & $\begin{array}{c}1-3 \\
4-11 \\
\geq 12\end{array}$ & $\begin{array}{l}0.118 \\
0.068 \\
0.082\end{array}$ & $\begin{array}{c}p_{1} \\
0.693 \\
0.438 \\
0.373\end{array}$ & $\begin{array}{l}0.921 \\
0.897 \\
0.842\end{array}$ \\
\hline Spiders & $\begin{array}{l}1-3 \\
4-11 \\
\geq 12\end{array}$ & $\begin{array}{l}0.242 \\
0.129 \\
0.115\end{array}$ & $\begin{array}{l}0.790 \\
0.645 \\
0.511\end{array}$ & $\begin{array}{l}0.935 \\
0.918 \\
0.884\end{array}$ \\
\hline
\end{tabular}

Notes. Columns are as follows: 1: sample. 2: group richness. 3-5: values of quantiles of galaxy properties (1st quantile, median, and 3rd quantile). 